\title{
Qing-Xin-Jie-Yu Granules in addition to conventional treatment for patients with stable coronary artery disease (QUEST Trial): study protocol for a randomized controlled trial
}

Shengyao Li ${ }^{1,2}$, Ming Guo ${ }^{1,2}$, Huimin Mao ${ }^{1,3}$, Zhuye Gao ${ }^{1}$, Hao X ${ }^{1 *}$ and Dazhuo Shi ${ }^{*}$

\begin{abstract}
Background: Recurrent cardiovascular event remains high in stable coronary artery disease (SCAD), especially in patients with multiple risk factors, despite a high rate of use conventional treatment. Traditional Chinese Medicine (TCM) is a promising complementary and alternative medicine for treating SCAD, while evidence for its effect on long-term survival is limited. This study was designed to test if Chinese herbal medicine in addition to conventional treatment is more effective than conventional treatment alone in reducing major adverse cardiac event (MACE) for SCAD patients with multiple risk factors during a 1-year follow-up.

Methods: This is a multicenter, placebo-controlled, double-blinded, randomized controlled clinical trial. A total of 1500 patients are randomized in a 1:1 ratio to receive the Qing-Xin-Jie-Yu Granules (QXJYG) or the placebo granules, twice daily for 6 months. The primary outcome is the combined outcomes including cardiac death, nonfatal myocardial infarction and revascularization. The secondary outcome is the combined outcomes including all-cause mortality, re-admission for acute coronary syndrome (ACS), heart failure, malignant supraventricular and ventricular arrhythmia influencing hemodynamics, ischemic stroke, and other thromboembolic events during 1-year follow-up. The assessment is performed at baseline (before randomization), 1, 3, 6, 9, and 12 months after randomization.
\end{abstract}

Discussion: This is the first multicenter trial sponsored by the national funding of China to evaluate TCM in combination with conventional treatment on 1-year survival in high-risk SCAD patients. If successful, it will provide an evidence-based complementary therapeutic approach for reducing MACE from SCAD.

Trial registration: The trial was registered in the Chinese Clinical Trial Registry on December 28, 2013. The registration number is ChiCTR-TRC-13004370.

Keywords: Stable coronary artery disease, Chinese herbal medicine, Qing-Xin-Jie-Yu Granules, Inflammation, Randomized controlled trial

\footnotetext{
*Correspondence: xuhaotcm@hotmail.com; shidazhuo@126.com

1 Department of Cardiology, Xiyuan Hospital of China Academy of Chinese

Medical Sciences, Beijing 100091, China

Full list of author information is available at the end of the article
} 


\section{Background}

Over the past three decades, preventive and therapeutic agents have substantially improved the prognosis of stable coronary artery disease (SCAD), however, cardiovascular event remains high, especially in high-risk patients with additional multiple risk factors, such as hypertension, hyperlipidemia and diabetes mellitus, despite a high rate of use conventional treatment including aspirin, statins, $\beta$-blockers and renin-angiotensinaldosterone system blockers, etc. [1]. In addition, increasing evidence supports a vital role of inflammation in the clinical instability of SCAD [2]. C-reactive protein (CRP), a systemic inflammatory marker, adds prognostic information to the cluster of traditional risk factors [3]. However, the novel anti-inflammatory drugs did not show a positive effect on further reducing major adverse cardiac event (MACE) [4, 5]. Therefore, it is necessary to seek more effective strategies for inhibiting inflammation and thus reducing the occurrence of MACE from SCAD.

Traditional Chinese Medicine (TCM) has been used to treat CAD for thousands of years. A meta-analysis of 70 randomized clinical trials including 9706 cases of CAD demonstrated a beneficial effect of TCM on clinical symptoms (odds ratio, 3.06 [95\% CI, 2.58 to 3.62]; $P<0.00001$ ) and electrocardiograph (odds ratio, 2.46 [95 \% CI, 2.16 to 2.80]; $P<0.00001$ ) compared with isosorbide dinitrate, and no serious adverse effects were reported [6]. However, another systematic review pointed out that the majority of randomized controlled trials on CAD with TCM are of "poor quality", thus more powerful evidence from highquality clinical trials are needed for future clinical practice [7].

Qing-Xin-Jie-Yu Granules (QXJYG) have been used to treat CAD for nearly two decades in mainland China, and previous small-sample studies have demonstrated that QXJYG relieved angina pectoris and decreased cholesterol of patients with CAD [8]. QXJYG consist of five herbal granules, including Astragalus membranaceus (Huangqi), Salvia miltiorrhiza Bunge (Danshen), Ligusticum chuanxiong Hort (Chuanxiong), Agastache rugosus (Huoxiang), and Coptis chinensis (Huanglian). The pharmacological studies suggested that the bioactive component or structural modification of extracts from Huangqi [9], Danshen [10], and Chuanxiong [11] had effects on cardiac protection, plaque stabilization, inhibition of platelet aggregation, etc., and these three Chinese herbs are also widely used in TCM for treating CAD [12-14]. Moreover, extracts from Danshen and Huanglian exert antiinflammatory effects $[15,16]$, and clinical studies showed that they decreased the level of high-sensitivity CRP (hs-CRP) in patients with cardiovascular disease $[17,18]$.
Therefore, QXJYG is worth investigating in high-risk SCAD patients due to the pleiotropic effect. The assumption is made that QXJYG in addition to conventional treatment may be superior to reduce inflammation of atherosclerosis and finally lead to the reduction of MACE in high-risk SCAD patients. If successful, it will provide an evidence-based complementary therapeutic approach for inhibiting inflammation and reducing MACE from SCAD.

\section{Methods/design \\ Study objectives}

This study is designed as a multicenter, double-blind, randomized, placebo-controlled, parallel-group, superiority trial to test the hypothesis that QXJYG in addition to conventional treatment is more effective than conventional treatment alone in reducing MACE, which is defined as cardiac death, nonfatal myocardial infarction and revascularization, for SCAD patients with two or more atherogenic risk factors during 1-year follow-up (see Fig. 1).

\section{Design overview}

The rigorous design, organization, and conduct of the trial are supervised by a Steering Committee, which comprises two members from each participating center in addition to the chairman, scientific coordinator and statistician. Eighteen clinical centers in mainland China will participate in the trial, including Xiyuan Hospital of China Academy of Chinese Medical Sciences, China-Japan Friendship Hospital, The First Hospital of Tianjin University of Traditional Chinese Medicine, etc. Data management and statistical analyses will be performed solely by data handlers and data analysts at Beijing Jiaotong University. This study follows the international recommendations for interventional trials [19]. (See the SPIRIT checklist in Additional file 1). All patients must personally sign and date an informed consent document before randomization.

\section{Inclusion criteria}

The trial will recruit patients of both genders aged 18-75 years with evidence of CAD documented by previous myocardial infarction ( $>3$ months before screening), percutaneous coronary revascularization ( $>1$ month before screening), angiographic or cardiac computed tomography angiographic evidence of $\geq 50 \%$ stenosis of $\geq 1$ major coronary artery. Also required are two or more of the following four risks: a serum hs-CRP level $\geq 3 \mathrm{mg} / \mathrm{L}$, a history of hypertension, hyperlipidemia, and diabetes mellitus.

\section{Exclusion criteria}

The exclusion criteria are as follows:

(1) Combined with congenital or rheumatic heart disease or severe heart failure; 


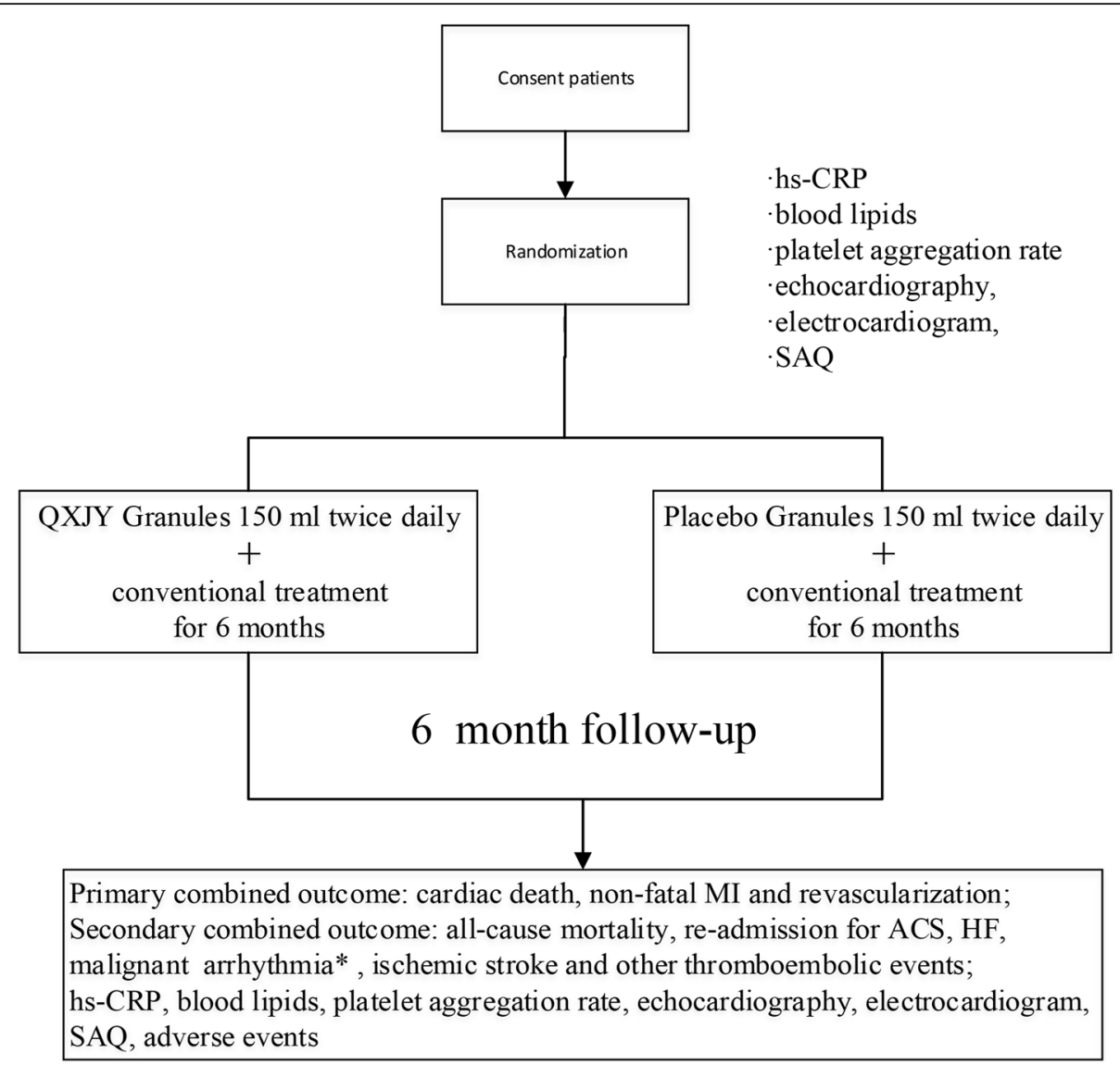

Fig. 1 Study flow of the QUEST Trial

(2) Uncontrolled severe arrhythmia (including paroxysmal ventricular tachycardia and supraventricular arrhythmia), which can cause hemodynamic responses;

(3) Acute cerebrovascular disease;

(4) Uncontrolled blood pressure, with a systolic blood pressure $\geq 160 \mathrm{mmHg}$ or a diastolic blood pressure $\geq 100 \mathrm{mmHg}$;

(5) Severe primary hepatic, renal, hematologic or mental disorders;

(6) Major head, chest or abdominal surgery within 4 weeks or bleeding tendency;

(7) Pregnant or lactating women;

(8) Suspected to be allergic to Chinese herbal medicine;

(9) Currently participating in another clinical trial.

\section{Recruitment}

Inpatients and outpatients with SCAD in each center will be screened. Each potentially eligible patient will be assessed by an attending physician for whether the patient should be recruited. The aim, procedures, and possible side effects of the study granules will be explained in detail to the patients; all patients will be asked to sign a written informed consent form before randomization.
Neither financial nor nonfinancial incentives will be provided to attending physicians and patients for enrolment.

\section{Randomization and treatment assignment}

Patients are randomized in a 1:1 ratio through a centrally controlled, computer-generated, site-stratified, block randomization schedule. The study granules are labeled with serial numbers, and each patient will be assigned the lowest number available at each participating center. All patients, care providers, attending physicians, laboratory staff and biostatisticians are blind to treatment assignment until the database has been locked. Randomization started on October, 2014. Enrolment of 1500 patients is expected to be completed in August 2016.

\section{Interventions}

Eligible patients will be allocated to receive QXJYG or placebo for 6 successive months, in addition to conventional treatment including antiplatelet, lipid-lowing, antihypertensive or antidiabetic therapy, etc., according to the guidelines [20]. The QXJYG and placebo granules were produced and packed in a single batch (Production batch number:1405001H) by China Resources Sanjiu 
Medical and Pharmaceutical Co., Ltd., Shenzhen, China., which has no conflict of interest relevant to this study. The test results of drug quality were consistent with the Chinese Medicine Standards of the State Food and Drug Administration (SFDA). The placebo granules are composed of $10 \%$ crude drug of QXJYG and $90 \%$ starch, which have the identical appearance, smell, and scent as the active treatment granules. Patients will be instructed to dissolve the granules in $150 \mathrm{~mL}$ hot water and take orally twice daily for 6 successive months. Any other Chinese herbal decoction or Chinese patent medicine for treating $\mathrm{CAD}$ is prohibited during the study.

\section{Outcomes}

The combined primary outcomes include cardiac death, nonfatal myocardial infarction, and revascularization. The combined secondary outcomes include all-cause mortality, re-admission for acute coronary syndrome (ACS), heart failure, malignant supraventricular and ventricular arrhythmia influencing hemodynamics, ischemic stroke, and other thromboembolic events during 1-year follow-up.

Hs-CRP, blood lipids, platelet aggregation rate, echocardiography, electrocardiogram, and Seattle Angina Questionnaire (SAQ) will also be monitored periodically.

\section{Safety assessment}

Safety will be monitored up to 6 months after intervention and evaluated based on the incidence of adverse events (AEs) including clinically significant changes in physical examinations, vital signs, and standard clinical laboratory tests (complete blood count, blood biochemical examination, coagulation [including prothrombin time (PT), thrombin time (TT), international normalized ratio (INR), activated partial thromboplastin time (APTT), and fibrinogen] and urinalysis). The National Cancer Institute's Common Terminology Criteria for Adverse Events (CTCAE) v4.0 grading system was used to classify the nature and severity of the AEs [21].

\section{Follow-up}

The follow-up for all patients will be scheduled at 1, 3, 6, 9, and 12 months (see Additional file 2). The primary and secondary outcomes as well as safety will be evaluated at each follow-up. If severe adverse events (SAEs) occur, the attending physician must report to the principal investigator and ethics committee immediately, who will decide whether or not to continue the treatment. Patients are also free to continue or discontinue the study at any time during the trial. Additionally, any medication change and events leading to therapy interruption will be recorded on the case record form (CRF).

\section{Compliance}

A mobile application will be used to remind the patients automatically at each follow-up appointment. In addition, each patient will be asked to offer at least two telephone numbers in order to be contacted in time. Furthermore, attending physicians will be responsible for each patient's questions.

To assess medication adherence, patients will be asked to return the unused granules. Unused granules accounting for more than $20 \%$ of the total granules will be defined as nonadherence. The reasons for nonadherence (e.g., discontinuation of intervention owing to harm or unsatisfactory efficacy) will be recorded on the CRF.

\section{Sample size}

The sample size was calculated based on the expected reduction of incidence in MACE from pre- to posttreatment at 1 year. A previous study suggested that the MACE (death, myocardial infarction, and urgent revascularization) incidence in high-risk patients with SCAD is about $10 \%$ within 1 year [1]. Assuming a MACE incidence reduction to $7 \%$ following treatment with QXJYG added, and given a type I error rate of alpha $=0.05$, a power of $80 \%$ (type II error rate of beta $=0.2$ ), the sample size for one arm needed to be 630 , resulting in $n=2 \times 630=1260$ patients. Considering a dropout rate of $20 \%$, a total of 1512 patients needed to be allocated to reach the required number of patients for the efficacy analysis. For convenience of randomization, we decided to recruit 1500 patients.

\section{Statistical approach}

Continuous variables will be presented as the mean \pm standard deviation (SD), median or interquartile range (IQR), and as frequencies or percentages for categorical variables. The comparability of the characteristics between the two groups will be assessed using a twosample Student's $t$ test for continuous variables and the chi-square test or Wilcoxon test, when appropriate, for categorical variables. For the SAQ scores, the analysis of covariance (ANCOVA) will be used to compare the change from baseline to 1 year between the QXJYG group and the placebo group [22]. Questionnaire score data, known as nonnormally distributed, will be transformed or analyzed with nonparametric ANCOVA. If appropriate, multivariable linear models will be used to assess the association between independent predictors and the continuous endpoints of the trial. Cumulative event rates of an outcome in the QXJYG and the placebo groups, respectively, will be estimated using the Kaplan-Meier method, and differences between the curves will be tested using the log-rank method. The crude and adjusted hazard ratio and their $95 \%$ confidence 
intervals will be estimated by the Cox proportional hazards regression model.

The primary outcome will be conducted on the intention-to-treat (ITT) analysis. The ITT set includes all patients randomized to treatment. The per protocol set consists of all patients with no major deviation from the protocol and with an overall treatment adherence rate of $80 \%$ or higher at the end of the study. The safety set consists of ITT patients excluding those who do not take any study medication or who have no record of follow-up after randomization. We will assess the effect that any missing data might have on the results by sensitivity analysis of augmented data sets. Dropouts will be included in the analysis by modern imputation methods for missing data.

We plan to conduct three subgroup analyses: first, we will compare hazard ratios of MACE based upon the level of hs-CRP (below $3 \mathrm{mg} / \mathrm{L}$ vs. greater than or equal to $3 \mathrm{mg} / \mathrm{L}$ ). Second, we will compare the hazard ratios of MACE between SCAD patients with diabetes or not. Finally, we will compare the hazard ratios of MACE between men and women with SCAD.

For all analyses, a value of $P<0.05$ is considered statistically significant, and all tests are two-tailed. $P$ values will be reported to four decimal places. All analyses will be conducted using R software version 2.15.1 (http://www.Rproject.org/), unless otherwise noted.

\section{Data management and monitoring}

Data from all participating centers will be imported in the clinical data management system (CDMS) with the website at http://www.xyedc.com. Data and materials supporting the conclusions of this trial will be available in this system. A password system will be utilized to control access. Data integrity will be achieved through several mechanisms including referential data rules, valid values, range checks and consistency checks against data already stored in the database. Missing data or specific errors in the data will be detected by programs. Written documentation of changes will be available via electronic logs and audit trails. Original CRF will be kept at the participating center for 5 years after completion of the study.

\section{Safety monitoring}

A Data and Safety Monitoring Board (DSMB) will be in charge of safety reviews, and will include at least two independent faculty members from the participating center. The safety will be evaluated for the first time when approximately $20 \%$ patients have 6 months of data available. Subsequently, the DSMB will perform the interim analysis for safety with the support of the statistical group when approximately $50 \%$ patients have 6 months of data available. In addition, the DSMB will conduct at least one on-site monitoring visit during the study to ensure that each participating center complies with the study protocol and Good Clinical Practice (GCP) principles; the data and SAEs are accurately and appropriately recorded on the CRF. When SAEs occur, the DSMB will perform unblinding immediately.

\section{Discussion}

According to our knowledge, this is the first multicenter trial sponsored by the national funding of China to evaluate TCM in combination with conventional treatment on 1-year survival in high-risk SCAD patients.

Atherogenic risk factors including hypertension, hyperlipidemia, and diabetes are common in CAD patients, and clustering of them has a higher age-adjusted, long-term mortality risk [23]. In addition, accumulating evidence has revealed that all these traditional atherogenic risk factors are closely associated with an increased level of CRP, an inflammatory marker with additional prognostic value [24]. A study showed that in SCAD patients, the CRP concentration in the fifth quintile $(>3.6 \mathrm{mg} / \mathrm{L})$ showed an about twofold increase in MACE, compared with the first four quintiles [25]. This is in accordance with the consensus view that inflammation has a role in the pathogenesis of cardiovascular events. Therefore, inhibiting chronic inflammation has been regarded a potential strategy to reduce MACE from SCAD.

Chinese herbs have shown multifactorial cardiovascular protective effects, such as lowing plasma lipids [9], reducing oxidative stress [10], and inhibiting platelet aggregation [11], etc. In addition, the anti-inflammatory effects of Chinese herbs have gained much attention recently. Danshen and Huanglian, the two herbs used in QXJYG, also have been demonstrated in many animal or cell culture models to inhibit inflammatory respondence. In ApoE-deficient mice fed with a high cholesterol diet, salvianolic acid B, an extract from Danshen, reduced metalloproteinases-2 (MMP-2) and MMP-9 expression significantly [26]. In human umbilical vein endothelial cells as well as platelets, treatment with extract from Danshen attenuated tumor necrosis factor alpha (TNF$\alpha$ )-induced expression of CD40. Furthermore, the expression of vascular cell adhesion molecule-1 (VCAM-1) and intercellular adhesion molecule-1 (ICAM-1) as well as the release of soluble VCAM-1, ICAM- 1 , interleukin6 (IL-6), IL-8 and monocyte chemoattractant protein 1 (MCP-1) were downregulated [27]. Berberine, an extract from Huanglian, also decreased serum levels of TNF- $\alpha$ and IL-6 in rats with isoproterenol-induced acute myocardial ischemia [28]. This gives a stimulus for large controlled trials to examine the long-term effects of QXJYG in CAD patients, especially those in a high 
inflammatory state (a serum hs-CRP level $\geq 3 \mathrm{mg} / \mathrm{L}$, with additional multiple atherogenic risk factors).

Granules have been widely used in mainland China. Our study QXJYG granules were produced strictly in compliance with standards of Good Manufactory Practice (GMP) and Chinese Pharmacopoeia 2010. The chemical compositions of the final products were analyzed for contamination with heavy metals, toxic elements, microbe and pesticide residues; the final products were analyzed for stability and adherence to quality standards of the CFDA. The fingerprint of QXJYG will be conducted to ensure the stability and homogeneity of the composition. The placebo granules have the identical appearance, smell, and scent as the active treatment granules. Granules were distributed to the 18 study sites with the same batch number. The company that provided the granules does not participate in the design, analysis, or interpretation of the study.

It should be noted that our study has limitations. First, the study is conducted only in mainland China, and whether the results from this trial can be applied to other ethnic groups is uncertain. However, the participating centers are located in different geographic areas in mainland China, and national minorities are also involved. Therefore, the recruited patients guarantee a certain representativeness. Second, the duration of follow-up is designed relatively short. The continuation of follow-up after the end of the study has been planned in order to assess the long-term outcome.

In conclusion, this trial is designed to demonstrate that the addition of QXJYG to conventional treatment will reduce the inflammation of atherosclerosis and finally lead to the reduction of MACE in high-risk SCAD patients.

\section{Trial status}

The trial has currently recruited 1202 patients since October 2014, of which 906 have completed the 6-month follow-up. Thirty patients experienced MACE, but their allocated group is still bind. No SAEs have occurred to date.

\section{Additional files}

Additional file 1: The SPIRIT Checklist. (DOC $123 \mathrm{~kb}$ )

Additional file 2: SPIRIT figure. (DOCX $23 \mathrm{~kb}$ )

\section{Abbreviations}

ACS: Acute coronary syndrome; AEs: Adverse events; APTT: Activated partial thromboplastin time; CAD: Coronary artery disease; CDMS: Clinical data management system; CRF: Case record form; CRP: C-reactive protein; DSMB: Data and Safety Monitoring Board; GCP: Good clinical practice; hs-CRP: High-sensitive C-reactive protein; ICAM-1: Intercellular adhesion molecule-1; IL-6: Interleukin-6; INR: International normalized ratio;
ITT: Intention-to-treat; MACE: Major adverse cardiac event; MCP-1: Monocyte chemoattractant protein-1; MMP-2: Metalloproteinases-2; PT: Prothrombin time; QXJYG: Qing-Xin-Jie-Yu Granules; SAEs: Severe adverse events; SAQ: Seattle Angina Questionnaire; SCAD: Stable coronary artery disease; TCM: Traditional Chinese Medicine; TNF-a: Tumor necrosis factor alpha; TT: Thrombin time; VCAM-1: Vascular cell adhesion molecule-1

\section{Funding}

This work is supported by grant 2013BAI02B01 from China's 12th Five-Year Plan. The funding source, China's Ministry of Science and Technology, had no role in the design of this study and will not have any role during its execution, analyses, interpretation of the data, or the decision to submit results.

\section{Authors' contributions}

DZS and HX were responsible for the design, the supervision of the study, and revision of the manuscript. SYL drafted the manuscript. MG, HMM, and ZYG participated in the revision of the manuscript and coordination of the study. All authors contributed to the refinement of the study protocol and approved the final manuscript.

\section{Competing interests}

The authors declare that they have no competing interests.

\section{Consent for publication}

We have gained informed consent for publication of the data set from patients at the point of recruitment to the trial. All the patients will be fully anonymous.

\section{Ethics approval and consent to participate}

The final amendments (version:2013-V-1, 23 December, 2013) and the consent form have been reviewed and approved by the Ethics Committee of Xiyuan Hospital of China Academy of Chinese Medical Sciences (reference number: 2013XL063-1). If there is any amendment to the protocol, approval must be again sought from the Ethics Committee. The study, registered in the Chinese Clinical Trial Registry (ChiCTR-TRC-13004370), complies with the principles of the Declaration of Helsinki and Good Clinical Practice guidelines.

\section{Dissemination policy}

The results will be disseminated regardless of the magnitude or direction of the effect after the study ends. Trial results will be published in peerreviewed journals, and presented at national and international scientific meetings.

\section{Author details}

1Department of Cardiology, Xiyuan Hospital of China Academy of Chinese Medical Sciences, Beijing 100091, China. ${ }^{2}$ Graduate School, China Academy of Chinese Medical Sciences, Beijing 100700, China. ${ }^{3}$ Graduate School, Beijing University of Chinese Medicine, Beijing 100029, China.

Received: 19 April 2016 Accepted: 23 August 2016

Published online: 15 September 2016

References

1. De Bruyne B, Pijls NHJ, Fearon WF. Fractional flow reserve-guided PCI in stable coronary disease. New Engl J Med. 2012:367:2356

2. Epstein FH, Ross R. Atherosclerosis_an inflammatory disease. New Engl J Med. 1999;340:115-26.

3. Ridker PM. Clinical application of C-reactive protein for cardiovascular disease detection and prevention. Circulation. 2003;107:363-9.

4. O'Donoghue ML, Braunwald E, White HD, Lukas MA, Tarka E, Steg PG, et al. Effect of darapladib on major coronary events after an acute coronary syndrome: the SOLID-TIMI 52 randomized clinical trial. JAMA. 2014;312:1006-15.

5. Nicholls SJ, Kastelein JJ, Schwartz GG, Bash D, Rosenson RS, Cavender MA, et al. Varespladib and cardiovascular events in patients with an acute coronary syndrome: the VISTA-16 randomized clinical trial. JAMA. 2014;311:252-62. 
6. Yao Y, Feng Y, Lin W. Systematic review and meta-analysis of randomized controlled trials comparing compound danshen dripping pills and isosorbide dinitrate in treating angina pectoris. Int J Cardiol. 2015;182:46-7.

7. Li WW, Guo H, Li HH, Wang LL, Fu H, Wang XM. Integration of traditional Chinese medicines and Western medicines for treating diabetes mellitus with coronary heart disease: a systematic review. J Altern Complement Med. 2013;19:492-500

8. Liao X, Luo LY. Clinical observation on Yu-Geng-Tong-Yu decoction in treating angina pectoris. Chin J Integr Med. 1998;18:594-7.

9. Chu C, Qi LW, Liu EH, Li B, Gao W, Li P. Radix Astragali (Astragalus): latest advancements and trends in chemistry, analysis, pharmacology and pharmacokinetics. Curr Org Chem. 2010;14:1792-807.

10. Gao S, Liu Z, Li H, Little PJ, Liu P, Xu S. Cardiovascular actions and therapeutic potential of tanshinone IIA. Atherosclerosis. 2012;220:3-10.

11. Chen H, Li G, Zhan P, Liu X. Ligustrazine derivatives. Part 5: design, synthesis and biological evaluation of novel ligustrazinyloxy-cinnamic acid derivatives as potent cardiovascular agents. Eur J Med Chem. 2011;46:5609-15.

12. Shang H, Zhang J, Yao C, Liu B, Gao X, Ren M, et al. Qi-shen-yi-qi dripping pills for the secondary prevention of myocardial infarction: a randomised clinical trial. Evid Based Complement Alternat Med. 2013;2013:738391.

13. Chen KJ, Shi DZ, Xu H, Lü SZ, Li TC, Ke YN, et al. XS0601 reduces the incidence of restenosis: a prospective study of 335 patients undergoing percutaneous coronary intervention in China. Chinese Med J. 2006;119:6-13.

14. Wang $S L$, Wang $C L$, Wang $P L$, Xu H, Liu HY, Du JP, et al. Combination of Chinese herbal medicines and conventional treatment versus conventional treatment alone in patients with acute coronary syndrome after percutaneous coronary intervention (5C Trial): an open-label randomized controlled, multicenter study. Evid Based Complement Alternat Med. 2013;2013:741518.

15. Cicero AF, Ertek S. Metabolic and cardiovascular effects of berberine: from preclinical evidences to clinical trial results. Clin Lipidol. 2009;4:553-63.

16. Ren Z, Tong $Y, X u$ W, Ma J, Chen Y. Tanshinone $\|$ A attenuates inflammatory responses of rats with myocardial infarction by reducing MCP-1 expression. Phytomedicine. 2010;17:212-8.

17. Xu GL, Zhao WX, Zhou ZM, Zhang RL, Zhu WS, Liu XF. Danshen extracts decrease blood C-reactive protein and prevent ischemic stroke recurrence: a controlled pilot study. Phytother Res. 2009;23:1721-5.

18. Chen H, Gao ZY, Xu H, Shi DZ, Chen KJ, Lv SZ, et al. Clinical study of unstable angina patients undergoing percutaneous coronary intervention treated with Chinese medicine for activating blood circulation and detoxicating. Chin J Integr Med Cardio-/Cerebrovasc Dis (Chin). 2009;10: 1135-7.

19. Chan A-W, Tetzlaff JM, Altman DG, Laupacis A, Gøtzsche PC, Krleža-Jerić K, Hróbjartsson A, Mann H, Dickersin K, Berlin J, Doré C, Parulekar W, Summerskill W, Groves T, Schulz K, Sox H, Rockhold FW, Rennie D, Moher D. SPIRIT 2013 Statement: defining standard protocol items for clinical trials. Ann Intern Med. 2013;158(3):200-7.

20. Chinese Society of Cardiology of Chinese Medical Association, Editorial Board of Chinese Journal of Cardiology. Guidelines for the diagnosis and management of chronic stable angina pectoris. Chinese J Cardiol. 2007;35:195-206.

21. Program NCICTE. Common Terminology Criteria for Adverse Events: (CTCAE): Cancer Therapy Evaluation Program. 2003.

22. Vickers AJ, Altman DG. Statistics notes: analysing controlled trials with baseline and follow-up measurements. BMJ. 2001;323:1123-4.

23. Lingman M, Herlitz J, Bergfeldt L, Karlsson T, Caidahl K, Hartford M. Acute coronary syndromes - the prognostic impact of hypertension, diabetes and its combination on long-term outcome. Int J Cardiol. 2009;137:29-36.

24. Ridker PM, Buring JE, Cook NR, Rifai N. C-reactive protein, the metabolic syndrome, and risk of incident cardiovascular events an 8-year follow-up of 14,719 initially healthy American women. Circulation. 2003;107:391-7.

25. Haverkate F, Thompson SG, Pyke SD, Gallimore JR, Pepys MB. Production of C-reactive protein and risk of coronary events in stable and unstable angina. European Concerted Action on Thrombosis and Disabilities Angina Pectoris Study Group. Lancet. 1997;349:462-6.

26. Lin SJ, Lee IT, Chen YH, Lin FY, Sheu LM, Ku HH, et al. Salvianolic acid B attenuates MMP-2 and MMP-9 expression in vivo in apolipoprotein-Edeficient mouse aorta and in vitro in LPS-treated human aortic smooth muscle cells. J Cell Biochem. 2007;100:372-84.
27. Stumpf C, Fan QL, Hintermann C, Raaz D, Kurfurst I, Losert S, et al. Anti-inflammatory effects of Danshen on human vascular endothelial cells in culture. Am J Chin Med. 2013;41:1065-77.

28. Zhang TZ, Yang SH, Du J. Protective effects of berberine on isoproterenolinduced acute myocardial ischemia in rats through regulating HMGB1-TLR4 axis. Evid-based Complement Altern Med. 2014;2014:8 pages.

\section{Submit your next manuscript to BioMed Central and we will help you at every step:}

- We accept pre-submission inquiries

- Our selector tool helps you to find the most relevant journal

- We provide round the clock customer support

- Convenient online submission

- Thorough peer review

- Inclusion in PubMed and all major indexing services

- Maximum visibility for your research

Submit your manuscript at www.biomedcentral.com/submit
Biomed Central 